\title{
Continuous Time Stochastic Models for Vehicular Traffic on Highways
}

\author{
Áttila L. Rodrigues and Mário J. de Oliveira \\ Instituto de Física, Universidade de São Paulo, Caixa Postal 66318, 05315-970, São Paulo, SP, Brazil
}

Received on 2 September, 2003

\begin{abstract}
We have simulated a continuous time version of the Nagel-Schreckenberger model of vehicular traffic on highways and calculated the flux as a function of vehicle density. In the low density regime the flux increases linearly with density but becomes a power law when velocities are allowed to increase without bounds. We have simulated also a modified version in which the state of a vehicle depends on the velocity of the vehicle moving ahead. This model displays a phase transition from a state with nonzero flux to a jammed state at a critical density which is strictly less than the closed-packed density. We also study the relationship of this model with self-organized criticality.
\end{abstract}

\section{Introduction}

Vehicular traffic flow is a subject, outside of the traditional areas of physics, that has been the object of investigation by the methods of statistical physics [1-7]. Stochastic nonequilibrium models defined on lattices are useful tools on the study of vehicular traffic from the microcopic point of view $[6,7]$. In this approach, the traffic of vehicles is modeled by a system of interacting particles, each particle representing an individual vehicle. The evolution of the system is defined by dynamical irreversible rules implying that these systems should be studied within the framework of nonequilibrium statistical mechanics [8-13]. We will be concerned here with the so called "particle-hoping" models. Originally, these models were defined as cellular automata, with deterministic rules, or as probabilistic cellular automata, with stochastic rules. In both cases the updating is synchronous. In this paper, however, we will examine the continuous time version of such models, with sequential updating or, more precisely, random sequential updating and whose time evolution of the probability distribution is governed by a master equation.

The stochastic microscopic models which we investigate here are defined by stochastic rules that are suitable for computer simulation. Numerical simulations are useful since anlytical approaches for nonequilibrium systems are very difficult to implement. Even though, we will present here some analytical results coming from mean-field approximations and compare them with numerical results. We will focus our attention on a continuous time version of the NagelSchreckenberger cellular automata model [14] of vehicular traffic on highways (model A) and a modification of it that takes into account the velocity of the vehicle moving ahead of another one (model B).

The main property we want to find is the relation between the flux and density, usually called the fundamental diagram of road traffic. For low densities, empiral data [15] shows a linear dependence of the flux on density. Increasing the density, the flux reachs a maximum (maximum capacity) and then drops. These properties are found for both models studied here. For model A, the flux vanishes when the density is the closed-packed density. However, for model B, the flux vanishes at a critical density which is strictly less than the closed-packed density.

\section{Model A}

Let us consider a system of $N$ particles moving in a one dimensional lattice with $L$ sites and periodic boudary conditions. Each site can be either empty or ocupied by a particle (vehicle). The particles move forwardly and may have any velocity up to a maximum velocity. The position and the velocity of the $j$ particle are denoted by $x_{j}$ and $v_{j}$, respectively. The distance between two consecutive sites is $a$ so that $x_{j}=a n_{j}$ where $n_{j}$ can take the values $0,1, \ldots, L-1$. The velocities of the particles take on discrete values $v_{j}=u \nu_{j}$ where $\nu_{j}=0,1, \ldots, \nu_{\mathrm{m}}$, so that the maximum velocity is $v_{\mathrm{m}}=u \nu_{\mathrm{m}}$. For simplicity we assume $a=1$ and $u=1$.

The state of the system is defined by the collection of the positions $\left\{x_{j}\right\}$ and velocites $\left\{v_{j}\right\}$ of the $N$ particles. The time evolution of the system is defined as follows. At each time interval $\tau / L$ one particle is chosen at random and its velocity and position are changed according to the following rules. The velocity is increased by one unity except if it has already the maximum velocity in which case it remains with the maximum velocity. The position of the particle is increased by an amount equal to the new velocity. In this model, however, there is no overtaking and two particles cannot occupy the same site. Therefore, one has to check whether there is enough space between the chosen particle and the particle moving ahead. If not, the new velocity will 
then be equal to the number of empty sites between the particle and the one ahead of it. Let $j$ be the chosen particle and let us denote the new velocity by $v_{j}^{\prime}$ and the new position by $x_{j}^{\prime}$. The rules are then

$$
v_{j}^{\prime}=\min \left\{\ell_{j}, v_{j}+1, v_{\mathrm{m}}\right\},
$$

where $\ell_{i}$ is the number of empty sites between the particle and the one that is moving ahead. The new position is

$$
x_{j}^{\prime}=x_{j}+v_{j}^{\prime} .
$$

We define the interval of time $\tau$ as the time it takes to make $N$ movements of particles or trials (a Monte Carlo step). The time step between two successive movement trials will be $\tau / N$. Since there are $N$ particles the average time between two movements of the same particle will be just $\tau$. Therefore, we must have the following relation $u=a \tau$.

The density of particles $\rho=N /(a L)$ is the number of particle per unit length. Since we are assuming $a=1$ then $\rho=N / L$. The fundamental property we want to analyze is the flux $\phi$ of particles as a function of the density $\rho$. The flux is the average number of particles crossing a certain point per unit time. If $M$ is the number of particle crossing a specified point of the lattice in the time interval $\tau$ then $\phi=M / \tau$. If we denote by $v$ the average velocity of particles given by

$$
v=\frac{1}{N} \sum_{j}\left\langle v_{j}\right\rangle
$$

then the flux is given by

$$
\phi=\rho v
$$

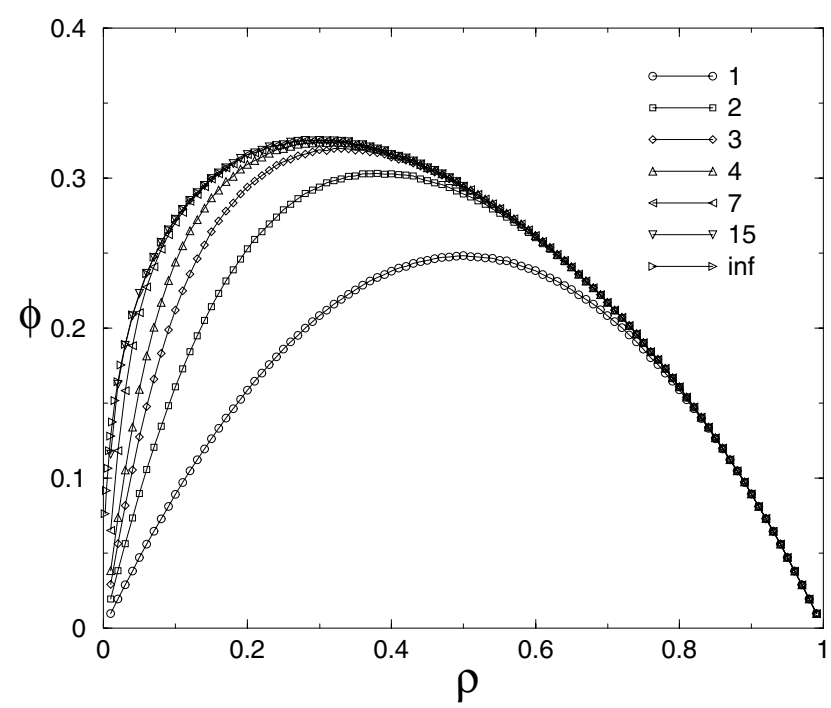

Figure 1. Flux $\phi$ versus density of vehicles $\rho$ for model A for several values of the maximum velocity $v_{\mathrm{m}}$ obtained from simulation of system with lattice size $L=1000$.

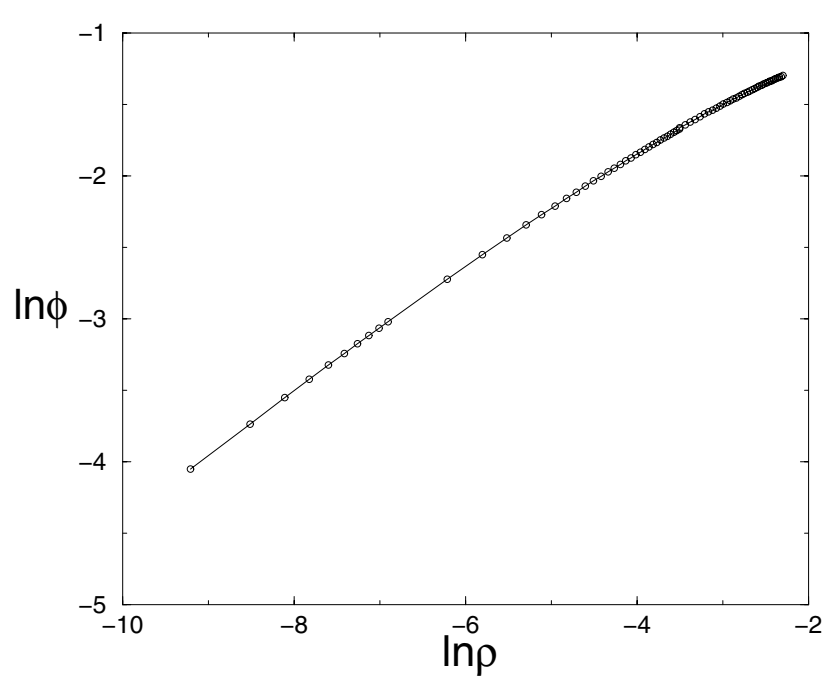

Figure 2. Log-log plot of the flux $\phi$ versus density of particles $\rho$ for $v_{\mathrm{m}}=\infty$ for model A obtained from simulation for a system with size $L=10^{5}$ A straight line fitted to the data points at small densities has slope 0.42 .

Using rules (1) and (2) we have simulated a system of $N$ particles with periodic boundary conditions with lattices with $L$ sites. Fig. 1 shows the flux of particles as a function of the density for $L=1000$ and for several values of the maximum velocity $v_{\mathrm{m}}$. The flux increases linearly at small densities, reaches a maximum and then decreases at high densities. For small densities the flux increases linearly according to

$$
\phi=v_{\mathrm{m}} \rho,
$$

as long as $v_{\mathrm{m}}$ is finite. If the maximum velocity is infinite, that is, if the velocity of a particle may increase without bounds, the behavior (5) is no longer valid. We assume then the following power law behavior for small densities

$$
\phi \sim \rho^{\alpha} .
$$

The double-log plot of $\phi$ versus $\rho$, shown in Fig. 2, gives the result $\alpha=0.42(1)$. In the high density regime, the flux becomes independent of the maximum velocity. For $\rho$ near 1 the flux behaves as

$$
\phi=1-\rho .
$$

To set up a mean-field approximation we assume that the stationary state is a non-correlated state. This means that, the probability that there is a gap of size $\ell$ between a given vehicle and the vehicle moving ahead is

$$
P_{\ell}=(1-\rho)^{\ell} \rho \text {. }
$$

We assume further that the velocity of the vehicle is $v_{\ell}=$ $\min \{v, \ell\}$. The average velocity will be then

$$
v=\sum_{\ell=0}^{\infty} v_{\ell} P_{\ell}=\frac{1-\rho}{\rho}\left\{1-(1-\rho)^{v_{\mathrm{m}}}\right\},
$$

which gives the flux

$$
\phi=\rho v=(1-\rho)\left\{1-(1-\rho)^{v_{\mathrm{m}}}\right\} .
$$

The mean-field approximantion given by (10) gives the general behavior expected for the flux and is correct at low and high densities as can be seen in Fig. 3. 


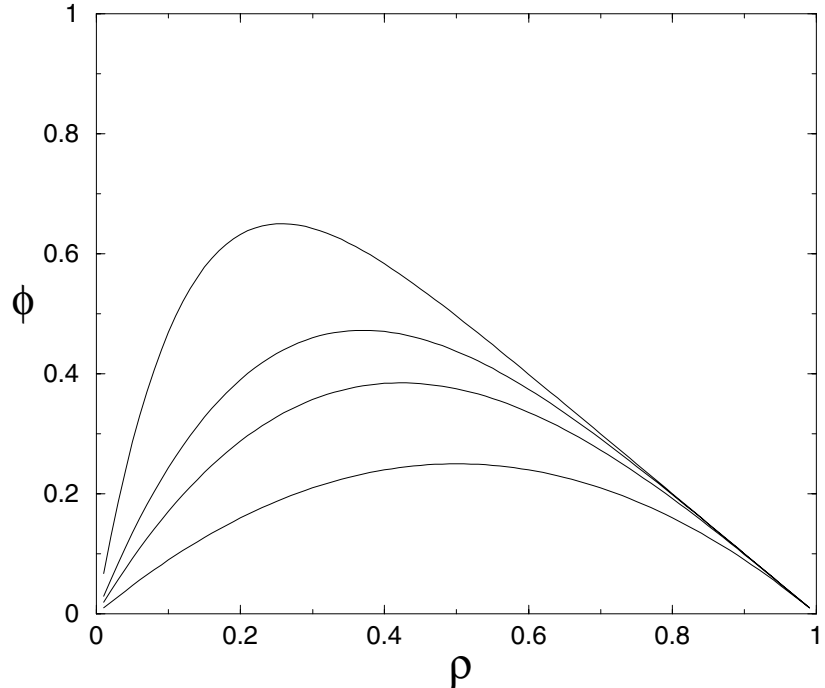

Figure 3. Flux $\phi$ versus density of vehicles $\rho$ for model A obtained from a mean-field approximation. The curves correspond, from botton to top, to $v_{\mathrm{m}}=1,2,3$, and 7 , respectively.

\section{Model B}

In this section we set up a model to describe more precisely the interaction between a vehicle, say vehicle $i$, and the vehicle which is moving ahead, vehicle $i+1$. In model A the velocity of the former does not depend on the velocity of the latter. In model $\mathrm{B}$, if the velocity of the vehicle $i+1$ is zero and the gap between vehicles $i$ and $i+1$ is 1 then the vehicle $i$ is not alowed to occupy the gap and it remains in its place with zero velocity. Therefore the rules are the same as those given by equations (1) and (2) except when $\ell_{i}=1$ in which case the new velocity is given by

$$
\begin{array}{lll}
v_{j}^{\prime}=0 & \text { if } & v_{i+1}=0, \\
v_{j}^{\prime}=1 & \text { if } & v_{i+1} \neq 0 .
\end{array}
$$

We have simulated model $\mathrm{B}$ for several values of the maximum velocity. The flux as a function of the density is shown in Fig. 4. At small densities the behavior is the same as the model A. However, at high density the behavior is entirely distinct from model A. As shown in Fig. 4, there is a critical density $\rho_{c}$ above which the flux vanishes. Table I shows the values of the critical density for several values of the maximum velosity $v_{\mathrm{m}}$. As one increases the density of vehicles, model B displays, therefore, a nonequilibrium phase transition from a state with nonzero flux to a jammed state with zero flux. The critical behavior of the flux near the transition is

$$
\phi \sim\left(\rho_{c}-\rho\right)^{\beta}
$$

with $\beta=1$.
TABLE 1: Critical density $\rho_{c}$ for several values of the maximum velocity of vehicles $v_{\mathrm{m}}$ for model $\mathrm{B}$.

\begin{tabular}{|l|l|}
\hline$v_{\mathrm{m}}$ & $\rho_{c}$ \\
\hline 1 & $0.582(1)$ \\
2 & $0.679(1)$ \\
3 & $0.768(1)$ \\
4 & $0.760(1)$ \\
7 & $0.768(1)$ \\
15 & $0.768(1)$ \\
$\infty$ & $0.768(1)$ \\
\hline
\end{tabular}

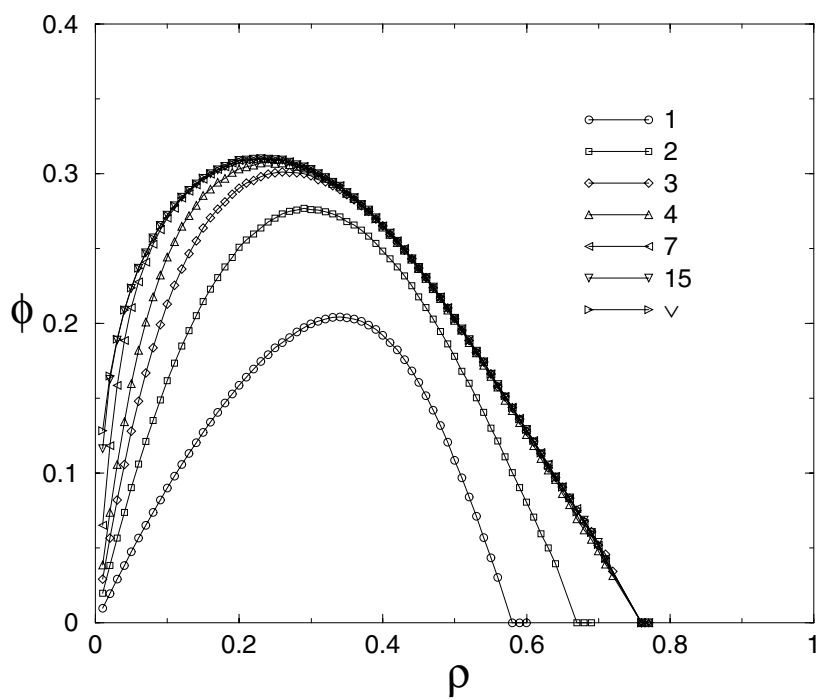

Figure 4. Flux $\phi$ versus density of vehicles $\rho$ for model B for several values of the maximum velocity $v_{\mathrm{m}}$ obtained from simulation of system with lattice size $L=1000$.

A jammed configuration is an absorbing state in which all cars have zero velocity. From the time evolution rules we see that any configuration of particles with zero velocity such that the gaps between particles are at most of size one is a jammed state. A jammed configuration may therefore occur whenever half of the sites are occupied so that there are infinitely many absorbing states. We expect then that the phase transition to the jammed state fall within the universality class of systems with infinitely many abosrbing states $[16,17]$. Since, the particles are driven to move in a given direction we expect a mean-field behavior and in particular $\beta=1$ which is the numerical result we have obtained here.

Another feature of the model B, and also of other models with infinitely many absorbing states, is its connection with sef-organized criticality. According to Dickman and collaborators [16] a system with infinitely many absorbing states may be interpreted as a self-organized system. In the present case of model B this relationship can be seen as follows. From a configuration with all sites occupied, we start by taking out of the system vehicles, chosen at random, until a configuration is reached such that the vehicles start to 
move. If after a certain number of Monte Carlo steps $T$, previously defined, the vehicles are still moving, we insert a vehicle into the system. If, on the other hand, the flux vanishes, before the maximum time $T$, we remove a vehicle, chosen at random. In other words, we remove a vehicle whenever the systems falls into a jammed state and insert one if the flux is nonzero. At the begining the density of vehicles $\rho$ in the system decreases linearly from the maximum value $\rho=1$ untill it reaches a value where it begins to fluctuates around a certain value $\rho^{*}$ of the density as can be seen in Fig. 5 for the case of system size $L=1000$ and maximum number of Monte Carlos steps $T=1000$. The inset of Fig. 5 shows that the density fluctuates around the value $\rho^{*}=0.731$.

The average density $\rho^{*}$ depends on the system size $L$ and on the maximum number of Monte Carlo steps $T$, and is expected to approach $\rho_{c}$ as $L \rightarrow \infty$ and $T \rightarrow \infty$.

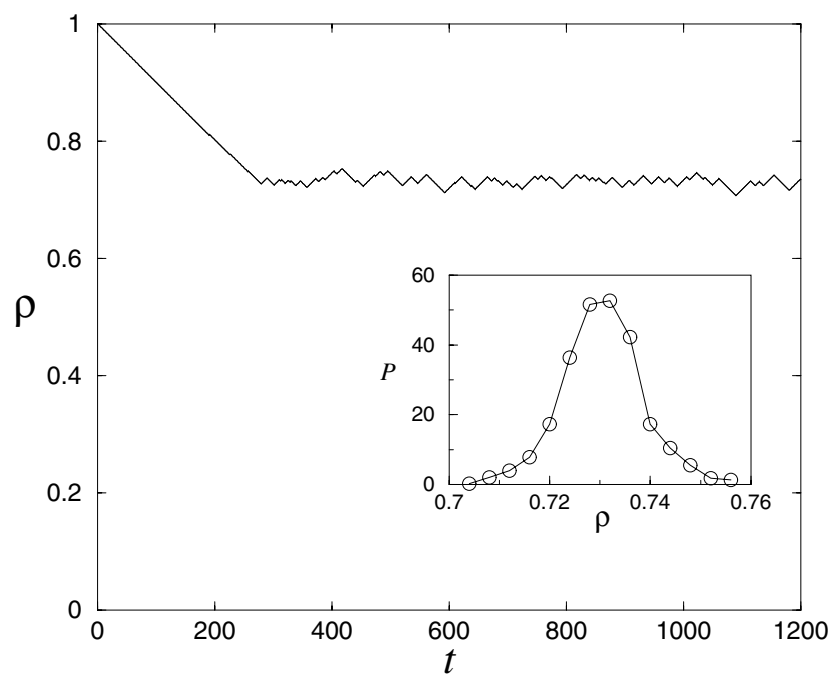

Figure 5. Density of particles for model B with infinity maximum velocity as a function of $t$, a quantity that increases by one unit whenever a particle is removed or added to the system. The inset shows a histogram of the densities with average $\rho^{*}=0.731$. The data are taken from a system with $L=1000$ and $T=1000$.

\section{Conclusion}

We have simulated two stochastic models for vehicular traffic on highways. In our approach, the traffic of vehicles is modeled by a system of interacting particles, each particle representing an individual vehicle. The first model is a continuous time version of the Nagel-Schreckenberger model. In the second we introduce an interaction between a vehicle and the vehicle moving ahead. We found that this second model has a phase transition from a state with nonzero flux to a jammed state which occurs for a density of particles wich is strictly less than the closed-packed density.

\section{Acknowledgements}

We wish to thak R. Dickman for helpful comments. We also acknowledge CNPq for finantial support.

\section{References}

[1] R. Herman and K. Gardels, Sci. Am. 209 (6), 35 (1963).

[2] F. A. Haight, Mathematical Theory of Traffic Flow (Academic Press, New York, 1963).

[3] W. D. Ashton, The Theory of Road Traffic Flow (Methuen, London, 1966).

[4] D. C. Gazis, Science 157, 273 (1967).

[5] I. Prigogine and R. Herman, Kinetic Theory of Vehicular Traffics (American Elsevier, New York, 1971).

[6] Traffic and Granular Flow (World Scientific, Singapore, 1996), edited by D. E. Wolf, M. Schreckenberg, and A. Bachem.

[7] D. Chowdhury, L. Santen, and A. Schadschneider, Physics Report 329, 199 (2000).

[8] T. M. Liggett, Interacting Particle Systems (Spinger-Verlag, New York, 1985).

[9] J. Marro and R. Dickman, Nonequilibrium Phase Transition in Lattice Models (Cambridge University Press, Cambridge, 1999).

[10] T. Tomé e M. J. de Oliveira, Dinâmica Estocástica e Irreversibilidade (Editora da Universidade de São Paulo, São Paulo, 2001).

[11] B. Schmittmann and R. K. P. Zia, in Phase Transitions and Critical Phenomena (Academic Press, New York, 1995), vol. 17, edited by C. Domb and J. L. Lebowitz.

[12] G. Schütz, in Phase Transitions and Critical Phenomena (Academic Press, New York, 2001), vol. 19, edited by C. Domb and J. L. Lebowitz.

[13] Nonequilibrium Statistical Mechanics in One Dimension (Cambridge University Press, Cambridge, 1997), Edited by V. Privman,

[14] K. Nagel and M. Schreckenberger, J. Physique I 2, 2221 (1992).

[15] F. L. Hall, B. L. Allen, and M. A. Gunter, Transp. Res. A 20, 197 (1986).

[16] R. Dickman, M. A. Muñoz, A. Vespignani, and S. Zapperi, Braz. J. Phys. 30, 27 (2000).

[17] R. Dickman, T. Tomé, and M. J. de Oliveira, Phys. Rev. E 66, 016111 (2002). 